POS $\quad$ PROCEEDINGS

\title{
Observable Gravitational Waves From Kinetically Modified Non-Minimal Inflation
}

\author{
C. Pallis* \\ Departament de Física Teòrica and IFIC, \\ Universitat de València-CSIC, \\ E-46100 Burjassot, SPAIN
}

\begin{abstract}
We consider Supersymmetric (SUSY) and non-SUSY models of chaotic inflation based on the simplest power-law potential with exponents $n=2$ and 4 . We propose a convenient non-minimal coupling to gravity and a non-minimal kinetic term which ensure, mainly for $n=4$, inflationary observables favored by the BICEP2/Keck Array and Planck results. Inflation can be attained for subplanckian inflaton values with the corresponding effective theories retaining the perturbative unitarity up to the Planck scale.
\end{abstract}

18th International Conference From the Planck Scale to the Electroweak Scale 25-29 May 2015

Ioannina, Greece

* Speaker. 


\section{Introduction}

Kinetically modified Non-minimal (chaotic) inflation (nMI) [1] is a variant of nMI which arises in the presence of a non-canonical kinetic term for the inflaton $\phi$ - apart from the non-minimal coupling $f_{R}(\phi)$ between $\phi$ and the Ricci scalar curvature, $R$ which is required by definition in any model of nMI [2]. In this talk we focus on inflationary models based on a synergy between $f_{R}$ and the inflaton potential $V_{\mathrm{CI}}$, which are selected $[1,3,4]$ as follows

$$
V_{\mathrm{CI}}(\phi)=\lambda^{2} \phi^{n} / 2^{n / 2} \text { and } f_{R}=1+c_{R} \phi^{n / 2} \text { with } n=2,4 \text {. }
$$

Below, we first (in Sec. ㅁ. briefly review the basic ingredients of nMI in a non-Supersymmetric (SUSY) framework and constrain the parameters of the models in Sec. [.3.3 taking into account a number of observational and theoretical requirements described in Sec. ‥2. Then (in Sec. ‥4) we focus on the problem with perturbative unitarity that plagues $[5,6]$ these models at the strong coupling and motivate the form of $f_{\mathrm{K}}$ analyzed in our work.

Throughout the text, the subscript, $\chi$ denotes derivation with respect to (w.r.t) the field $\chi$, charge conjugation is denoted by a star $\left(^{*}\right)$ and we use units where the reduced Planck scale $m_{\mathrm{P}}=$ $2.43 \cdot 10^{18} \mathrm{GeV}$ is set equal to unity.

\subsection{Coupling non-Minimally the Inflaton to Gravity}

The action of the inflaton $\phi$ in the Jordan frame (JF), takes the form:

$$
\mathrm{S}=\int d^{4} x \sqrt{-\mathfrak{g}}\left(-\frac{f_{R}}{2} R+\frac{f_{\mathrm{K}}}{2} g^{\mu v} \partial_{\mu} \phi \partial_{\nu} \phi-V_{\mathrm{CI}}(\phi)\right) .
$$

where $\mathfrak{g}$ is the determinant of the background Friedmann-Robertson-Walker metric, $g^{\mu v}$ with signature $(+,-,-,-),\left\langle f_{R}\right\rangle \simeq 1$ to guarantee the ordinary Einstein gravity at low energy and we allow for a kinetic mixing through the function $f_{\mathrm{K}}(\phi)$. By performing a conformal transformation [3] according to which we define the Einstein frame (EF) metric $\widehat{g}_{\mu \nu}=f_{R} g_{\mu v}$ we can write $\mathrm{S}$ in the EF as follows

$$
\mathrm{S}=\int d^{4} x \sqrt{-\widehat{\mathfrak{g}}}\left(-\frac{1}{2} \widehat{R}+\frac{1}{2} \widehat{g}^{\mu v} \partial_{\mu} \widehat{\phi} \partial_{\nu} \widehat{\phi}-\widehat{V}_{\mathrm{CI}}(\widehat{\phi})\right)
$$

where hat is used to denote quantities defined in the EF. We also introduce the EF canonically normalized field, $\widehat{\phi}$, and potential, $\widehat{V}_{\mathrm{CI}}$, defined as follows:

$$
\frac{d \widehat{\phi}}{d \phi}=J=\sqrt{\frac{f_{\mathrm{K}}}{f_{R}}+\frac{3}{2}\left(\frac{f_{R, \phi}}{f_{R}}\right)^{2}} \text { and } \widehat{V}_{\mathrm{CI}}=\frac{V_{\mathrm{CI}}}{f_{R}^{2}},
$$

where the symbol , $\phi$ as subscript denotes derivation w.r.t the field $\phi$. Plugging Eq. (메) into Eq. (ㄴ.3b), we obtain

$$
J^{2}=\frac{f_{\mathrm{K}}}{f_{R}}+\frac{3 n^{2} c_{R}^{2} \phi^{n-2}}{8 f_{R}^{2}} \quad \text { and } \quad \widehat{V}_{\mathrm{CI}}=\frac{\lambda^{2} \phi^{n}}{2^{n / 2} f_{R}^{2}}
$$

In the pure nMI [2-4] we take $f_{\mathrm{K}}=1$ and, for $c_{R} \gg 1$, we infer from Eq. ([L.3b), that $f_{R}$ determines the relation between $\widehat{\phi}$ and $\phi$ and controls the shape of $\widehat{V}_{\mathrm{CI}}$ affecting thereby the observational predictions - see below. 


\subsection{Inflationary Observables - Constraints}

A model of nMI can be qualified as successful, if it can become compatible with the following observational and theoretical requirements:

(i) The number of e-foldings $\widehat{N}_{\star}$ that the scale $k_{\star}=0.05 / \mathrm{Mpc}$ experiences during this nMI must to be enough for the resolution of the horizon and flatness problems of standard Big Bang, i.e., [7]

$$
\widehat{N}_{\star}=\int_{\widehat{\phi}_{\mathrm{f}}}^{\widehat{\phi}_{\star}} d \widehat{\phi} \frac{\widehat{V}_{\mathrm{CI}}}{\widehat{V}_{\mathrm{CI}, \widehat{\phi}}} \simeq 55,
$$

where $\phi_{\star}\left[\widehat{\phi}_{\star}\right]$ are the value of $\phi[\widehat{\phi}]$ when $k_{\star}$ crosses the inflationary horizon. Also $\phi_{\mathrm{f}}\left[\widehat{\phi}_{\mathrm{f}}\right]$ is the value of $\phi[\widehat{\phi}]$ at the end of nMI, which can be found, in the slow-roll approximation, from the condition

$$
\begin{gathered}
\max \left\{\widehat{\varepsilon}\left(\phi_{\mathrm{f}}\right),\left|\widehat{\eta}\left(\phi_{\mathrm{f}}\right)\right|\right\}=1 \text {, where } \\
\widehat{\varepsilon}=\frac{1}{2}\left(\frac{\widehat{V}_{\mathrm{CI}, \widehat{\phi}}}{\widehat{V}_{\mathrm{CI}}}\right)^{2}=\frac{1}{2 J^{2}}\left(\frac{\widehat{V}_{\mathrm{CI}, \phi}}{\widehat{V}_{\mathrm{CI}}}\right)^{2} \text { and } \widehat{\eta}=\frac{\widehat{V}_{\mathrm{CI}, \widehat{\phi} \widehat{\phi}}}{\widehat{V}_{\mathrm{CI}}}=\frac{1}{J^{2}}\left(\frac{\widehat{V}_{\mathrm{CI}, \phi \phi}}{\widehat{V}_{\mathrm{CI}}}-\frac{\widehat{V}_{\mathrm{CI}, \phi}}{\widehat{V}_{\mathrm{CI}}} \frac{J_{, \phi}}{J}\right) .
\end{gathered}
$$

It is evident from the formulas above that non trivial modifications on $f_{\mathrm{K}}$ and thus to $J$ may have an pronounced impact on the parameters above modifying thereby the inflationary observables too.

(ii) The amplitude $A_{\mathrm{s}}$ of the power spectrum of the curvature perturbation generated by $\phi$ at $k_{\star}$ has to be consistent with data [7], i.e.,

$$
\sqrt{A_{\mathrm{s}}}=\frac{1}{2 \sqrt{3} \pi} \frac{\widehat{V}_{\mathrm{CI}}\left(\widehat{\phi}_{\star}\right)^{3 / 2}}{\left|\widehat{V}_{\mathrm{CI}, \widehat{\phi}}\left(\widehat{\phi}_{\star}\right)\right|}=\frac{1}{2 \pi} \sqrt{\frac{\widehat{V}_{\mathrm{CI}}\left(\phi_{\star}\right)}{6 \widehat{\varepsilon}_{\star}}} \simeq 4.627 \cdot 10^{-5},
$$

where the variables with subscript $\star$ are evaluated at $\phi=\phi_{\star}$.

(iii) The remaining inflationary observables (the spectral index $n_{\mathrm{s}}$, its running $a_{\mathrm{s}}$, and the tensorto-scalar ratio $r$ ) - estimated through the relations:

$$
\text { (a) } n_{\mathrm{s}}=1-6 \widehat{\varepsilon}_{\star}+2 \widehat{\eta}_{\star}, \quad \text { (b) } a_{\mathrm{s}}=\frac{2}{3}\left(4 \widehat{\eta}^{2}-\left(n_{\mathrm{s}}-1\right)^{2}\right)-2 \widehat{\xi}_{\star} \text { and } \quad \text { (c) } r=16 \widehat{\varepsilon}_{\star},
$$

with $\widehat{\xi}=\widehat{V}_{\mathrm{CI}, \widehat{\phi}} \widehat{V}_{\mathrm{CI}, \widehat{\phi} \hat{\phi} \hat{\phi}} / \widehat{V}_{\mathrm{CI}}^{2}-$ have to be consistent with the data [7], i.e.,

$$
\text { (a) } n_{\mathrm{s}}=0.968 \pm 0.009 \text { and (b) } r \leq 0.12 \text {, }
$$

at $95 \%$ confidence level (c.l.) - pertaining to the $\Lambda \mathrm{CDM}+r$ framework with $\left|a_{\mathrm{s}}\right| \ll 0.01$. Although compatible with Eq. (파) the present combined Planck and BICEP2/Keck Array results [8] seem to favor $r$ 's of order 0.01 since $r=0.048_{-0.032}^{+0.035}$ at $68 \%$ c.l. has been reported.

(iv) The effective theory describing $\mathrm{nMI}$ has to remains valid up to a UV cutoff scale $\Lambda_{\mathrm{UV}}$ to ensure the stability of our inflationary solutions, i.e.,

$$
\text { (a) } \widehat{V}_{\mathrm{CI}}\left(\phi_{\star}\right)^{1 / 4} \leq \Lambda_{\mathrm{UV}} \text { and (b) } \phi_{\star} \leq \Lambda_{\mathrm{UV}} \text {. }
$$

It is expected that $\Lambda_{\mathrm{UV}} \simeq m_{\mathrm{P}}$, contrary to the pure nMI with $c_{R} \gg 1$ where $\Lambda_{\mathrm{UV}} \ll m_{\mathrm{P}}-$ see Sec. प.4. 


\subsection{The Two Regimes of Synergistic nMI}

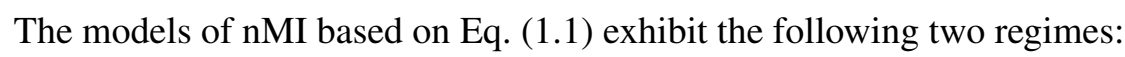

(i) The weak $c_{R}$ regime with $c_{R} \ll 1$. In this case from Eq. (ㄸ.3D) we find $J \simeq 1 / f_{R}$ and applying Eqs. (ㄷ.5) and (ㄸ.6), the slow-roll parameters and $\widehat{N}_{\star}$ read

$$
\widehat{\varepsilon} \simeq \frac{n^{2}}{2 \phi^{2} f_{R}}, \widehat{\eta} \simeq 2\left(1-\frac{1}{n}\right) \widehat{\varepsilon}-\frac{4+n}{2 n} c_{R} \phi^{\frac{n}{2}} \widehat{\varepsilon} \quad \text { and } \quad \widehat{N}_{\star} \simeq \frac{\phi_{\star}^{2}}{2 n} .
$$

Imposing the condition of Eq. ([ए]) and solving then the latter equation w.r.t $\phi_{*}$ we arrive at

$$
\phi_{\mathrm{f}} \simeq n / \sqrt{2} \text { and } \phi_{\star} \simeq \sqrt{2 n \widehat{N}_{\star}} .
$$

Inflation is attained, thus, only for $\phi>1$. On the other hand, Eq. (ㅍ.]) implies

$$
\lambda=\sqrt{6 A_{\mathrm{s}} f_{n \star}} \pi n^{(2-n) / 4} / \widehat{N}_{\star}^{(2+n) / 4},
$$

where $f_{n \star}=f_{R}\left(\phi_{\star}\right)=1+c_{R}\left(2 n \widehat{N}_{\star}\right)^{n / 4}$. Applying Eq. (ㄸ.8) we find that the inflationary observables are $c_{R}$-dependent and can be marginally consistent with Eq. (ㅁ. $)$ - see Sec. 13.2. Indeed,

$$
\begin{gathered}
n_{\mathrm{s}}=1-\left(4+n+n / f_{n \star}\right) / 4 \widehat{N}_{\star}, r=4 n / f_{n \star} \widehat{N}_{\star}, \\
a_{\mathrm{s}}=\left(n^{2}-n(n+4) f_{n \star}-4(n+4) f_{n \star}^{2}\right) / 16 f_{n \star}^{2} \widehat{N}_{\star}^{2} .
\end{gathered}
$$

In the limit $c_{R} \rightarrow 0$ or $f_{n \star} \rightarrow 1$ the results of the simplest power-law chaotic inflation - with $f_{R}=$ $f_{\mathrm{K}}=1$ and $V_{\mathrm{CI}}$ given in Eq. (ㅍ. $)$ - are recovered. These are by now disfavored by Eq. (ㄷ.9).

(ii) The strong $c_{R}$ regime with $c_{R} \gg 1$. In this case, from Eq. (प.3b) we find

$$
J \simeq \sqrt{3} n c_{R} \phi^{n / 2-1} / 2 \sqrt{2} f_{R} \text { and } \widehat{V}_{\mathrm{CI}} \simeq \lambda^{2} / 2^{n / 2} c_{R}^{2} .
$$

We observe that $\widehat{V}_{\mathrm{CI}}$ exhibits an almost flat plateau. From Eqs. ([L.J) and ([L.6) we find

$$
\widehat{\varepsilon} \simeq 4 / 3 c_{R}^{2} \phi^{n}, \widehat{\eta} \simeq-4 / 3 c_{R} \phi^{n / 2} \text { and } \widehat{N}_{\star} \simeq 3 c_{R} \phi_{*}^{n / 2} / 4 .
$$

Therefore, $\phi_{\mathrm{f}}$ and $\phi_{\star}$ are found from the condition of Eq. ([ए.]) and the last equality above, as follows

$$
\phi_{\mathrm{f}}=\max \left\{\left(4 / 3 c_{R}^{2}\right)^{1 / n},\left(4 / 3 c_{R}\right)^{2 / n}\right\} \quad \text { and } \quad \phi_{\star}=\left(4 \widehat{N}_{\star} / 3 c_{R}\right)^{2 / n} .
$$

Consequently, nMI can be achieved even with subplanckian $\phi$ values for $c_{R} \gtrsim\left(4 \widehat{N}_{*} / 3\right)^{2 / n}$. Also the normalization of Eq. (ㅍ. I) implies the following relation between $c_{R}$ and $\lambda$

$$
\left.A_{\mathrm{s}}^{1 / 2} \simeq 2^{-(10+n) / 4} \frac{\lambda c_{R} \phi^{n}}{\pi f_{R}}\right|_{\phi=\phi_{\star}} \Rightarrow \lambda \simeq \frac{3 \cdot 2^{n / 4}}{\widehat{N}_{\star}} \sqrt{2 A_{\mathrm{s}}} \pi c_{R} .
$$

From Eq. ([1.8) we obtain the $c_{R}$-independent values for the observables:

$$
n_{\mathrm{s}} \simeq 1-2 / \widehat{N}_{\star} \simeq 0.965, a_{\mathrm{s}} \simeq-2 / \widehat{N}_{\star}^{2} \simeq-6.4 \cdot 10^{-4} \text { and } r \simeq 12 / \widehat{N}_{\star}^{2} \simeq 4 \cdot 10^{-3},
$$

which are in agreement with Eq. (ㅍ.9), although with low enough $r$ values. 


\subsection{The Ultraviolet (UV) Cut-off Scale}

In the highly predictive regime with large $c_{\mathrm{K}}$, the models violate perturbative unitarity for $n>2$. To see this we analyze the small-field behavior of the theory in order to extract the UV cutoff scale $\Lambda_{\mathrm{UV}}$. The result depends crucially on the value of $J$ in Eq. ([L.36) in the vacuum, $\langle\phi\rangle=0$. Namely we have

$$
\langle J\rangle= \begin{cases}\sqrt{3 / 2} c_{R} & \text { for } n=2 \\ 1 & \text { for } n \neq 2\end{cases}
$$

For $n=2$ and any $c_{R}$ we obtain $\widehat{\phi} \neq \phi$. Expanding the second and third term of $\mathrm{S}$ in the right-hand side of Eq. ([L.3a) about $\langle\phi\rangle=0$ in terms of $\widehat{\phi}$ we obtain:

$$
J^{2} \dot{\phi}^{2}=\left(1-\sqrt{\frac{8}{3}} \widehat{\phi}+2 \widehat{\phi}^{2}-\cdots\right) \dot{\hat{\phi}}^{2} \text { and } \widehat{V}_{\mathrm{CI}}=\frac{\lambda^{2} \widehat{\phi}^{2}}{3 c_{R}^{2}}\left(1-\sqrt{\frac{8}{3}} \widehat{\phi}+2 \widehat{\phi}^{2}-\cdots\right) .
$$

As a consequence $\Lambda_{\mathrm{UV}}=m_{\mathrm{P}}$ since the expansions above are $c_{R}$ independent. On the contrary, for $n>2$ we have $\widehat{\phi}=\phi$ and the expansions of the same terms in Eq. (ㄴ.3al) are $c_{R}$ dependent:

$$
\begin{aligned}
& J^{2} \dot{\phi}^{2}=\left(1-c_{R} \widehat{\phi}^{\frac{n}{2}}+\frac{3 n^{2}}{8} c_{R}^{2} \widehat{\phi}^{n-2}+c_{R}^{2} \widehat{\phi}^{n}-\cdots\right) \dot{\hat{\phi}}^{2} ; \\
& \widehat{V}_{\mathrm{CI}}=\frac{\lambda^{2} \widehat{\phi}^{n}}{2}\left(1-2 c_{R} \widehat{\phi}^{\frac{n}{2}}+3 c_{R}^{2} \widehat{\phi}^{n}-4 c_{R}^{3} \widehat{\phi}^{\frac{3 n}{2}}+\cdots\right) .
\end{aligned}
$$

Since the term which yields the smallest denominator for $c_{R}>1$ is $3 n^{2} c_{R}^{2} \widehat{\phi}^{n-2} / 8$ we find [5,6]:

$$
\Lambda_{\mathrm{UV}}=m_{\mathrm{P}} / c_{R}^{2 /(n-2)} \ll m_{\mathrm{P}} .
$$

However, if we introduce a non-canonical kinetic mixing of the form

$$
f_{\mathrm{K}}(\phi)=c_{\mathrm{K}} f_{R}^{m} \quad \text { where } \quad c_{\mathrm{K}}=\left(c_{R} / r_{R \mathrm{~K}}\right)^{4 / n} \text { and } m \geq 0,
$$

no problem with the perturbative unitarity emerges for $r_{R \mathrm{~K}} \leq 1$, even if $c_{R}$ and/or $c_{\mathrm{K}}$ are large the latter situation is expected if we wish to achieve efficient nMI with $\phi \leq 1$. E.g., for $m=0$ the

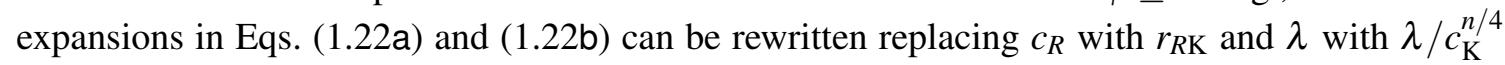
- similar expressions can be obtained for other $m$, too. In other words, the perturbative unitarity can be preserved up to $m_{\mathrm{P}}$ if we select a non-trivial $f_{\mathrm{K}}$ such that $\langle J\rangle \neq 1$. This requirement lets a functional uncertainty as regards the form of $f_{\mathrm{K}}$ during $\mathrm{nMI}$ which can be parameterized as shown in Eq. (‥24) given that $\left\langle f_{R}\right\rangle \simeq 1-$ see Sec. ‥

We below describe a possible formulation of this type of nMI in the context of Supergravity (SUGRA) - see Sec. \- and we then analyze the inflationary behavior of these models in Sec. B]. We conclude summarizing our results in Sec. . .

\section{Supergravity Embeddings}

The models above - defined by Eqs. (미) and (‥24) - can be embedded in SUGRA if we use two gauge singlet chiral superfields $z^{\alpha}=\Phi, S$, with $\Phi(\alpha=1)$ and $S(\alpha=2)$ being the inflaton and a "stabilizer" field respectively. The EF action for $z^{\alpha}$ 's can be written as [9]

$$
\mathrm{S}=\int d^{4} x \sqrt{-\widehat{\mathfrak{g}}}\left(-\frac{1}{2} \widehat{R}+K_{\alpha \bar{\beta}} \widehat{g}^{\mu v} \partial_{\mu} z^{\alpha} \partial_{v} z^{* \bar{\beta}}-\widehat{V}\right),
$$


where summation is taken over the scalar fields $z^{\alpha}, K$ is the Kähler potential with $K_{\alpha \bar{\beta}}=K_{, z^{\alpha} z^{* \bar{\beta}}}$ and $K^{\alpha \bar{\beta}} K_{\bar{\beta} \gamma}=\delta_{\gamma}^{\alpha}$. Also $\widehat{V}$ is the EF F-term SUGRA potential given by

$$
\widehat{V}=e^{K}\left(K^{\alpha \bar{\beta}} D_{\alpha} W D_{\bar{\beta}}^{*} W^{*}-3|W|^{2}\right),
$$

where $D_{\alpha} W=W_{, z^{\alpha}}+K_{, z^{\alpha}} W$ with $W$ being the superpotential. Along the inflationary track determined by the constraints

$$
S=\Phi-\Phi^{*}=0, \text { or } s=\bar{s}=\theta=0
$$

if we express $\Phi$ and $S$ according to the parametrization

$$
\Phi=\phi e^{i \theta} / \sqrt{2} \text { and } S=(s+i \bar{s}) / \sqrt{2},
$$

$V_{\mathrm{CI}}$ in Eq. (II.]) can be produced, in the flat limit, by

$$
W=\lambda S \Phi^{n / 2} .
$$

\begin{tabular}{|l||ll|}
\hline SUPERFIELDS: & $S$ & $\Phi$ \\
\hline \hline$U(1)_{R}$ & 1 & 0 \\
$U(1)$ & -1 & $2 / n$ \\
\hline
\end{tabular}

Table 1: Charge assignments of the superfields.

The form of $W$ can be uniquely determined if we impose an $R$ and a global $U(1)$ symmetry with charge assignments shown in Table $\mathbb{D}$.

On the other hand, the derivation of $\widehat{V}_{\mathrm{CI}}$ in Eq. (ㅁ.4) via Eq. (2.]W) requires a judiciously chosen $K$. Namely, along the track in Eq. (2.2) the only surviving term in Eq. (2.JW) is

$$
\widehat{V}_{\mathrm{CI}}=\widehat{V}(\boldsymbol{\theta}=s=\bar{s}=0)=e^{K} K^{S S^{*}}\left|W_{, S}\right|^{2} .
$$

The incorporation $f_{R}$ in Eq. (메) and $f_{\mathrm{K}}$ in Eq. (‥24) dictates the adoption of a logarithmic $K$ [9] including the functions

$$
F_{R}(\Phi)=1+2^{\frac{n}{4}} \Phi^{\frac{n}{2}} c_{R}, F_{\mathrm{K}}=\left(\Phi-\Phi^{*}\right)^{2} \quad \text { and } \quad F_{S}=|S|^{2}-k_{S}|S|^{4} .
$$

Here, $F_{R}$ is an holomorphic function reducing to $f_{R}$, along the path in Eq. (2.2),$F_{\mathrm{K}}$ is a real function which assists us to incorporate the non-canonical kinetic mixing generating by $f_{\mathrm{K}}$ in Eq. (ㄴ.24), and $F_{S}$ provides a typical kinetic term for $S$, considering the next-to-minimal term for stability/heaviness reasons [9]. Indeed, $F_{\mathrm{K}}$ lets intact $\widehat{V}_{\mathrm{CI}}$, since it vanishes along the trajectory in Eq. (․2]), but it contributes to the normalization of $\Phi$. Taking for consistency all the possible terms up to fourth order, $K$ is written as

$$
K_{1}=-3 \ln \left(\frac{1}{2}\left(F_{R}+F_{R}^{*}\right)+\frac{c_{\mathrm{K}}}{3 \cdot 2^{m+1}}\left(F_{R}+F_{R}^{*}\right)^{m} F_{\mathrm{K}}-\frac{1}{3} F_{S}+\frac{k_{\Phi}}{6} F_{\mathrm{K}}^{2}-\frac{k_{S \Phi}}{3} F_{\mathrm{K}}|S|^{2}\right) .
$$

Alternatively, if we do not insist on a pure logarithmic $K$, we could also adopt the form

$$
K_{2}=-3 \ln \left(\frac{1}{2}\left(F_{R}+F_{R}^{*}\right)-\frac{1}{3} F_{S}\right)-\frac{c_{\mathrm{K}}}{2^{m}} \frac{F_{\mathrm{K}}}{\left(F_{R}+F_{R}^{*}\right)^{1-m}} .
$$

Moreover, if we place $F_{S}$ outside the argument of the logarithm similar results are obtained by the following $K$ 's - not mentioned in Ref. [1]:

$$
\begin{aligned}
& K_{3}=-2 \ln \left(\frac{1}{2}\left(F_{R}+F_{R}^{*}\right)+\frac{c_{\mathrm{K}}}{2^{m+2}}\left(F_{R}+F_{R}^{*}\right)^{m} F_{\mathrm{K}}\right)+F_{S}, \\
& K_{4}=-2 \ln \frac{F_{R}+F_{R}^{*}}{2}-\frac{c_{\mathrm{K}}}{2^{m}} \frac{F_{\mathrm{K}}}{\left(F_{R}+F_{R}^{*}\right)^{1-m}}+F_{S} .
\end{aligned}
$$




\begin{tabular}{|c|c|c|c|c|c|}
\hline \multirow{2}{*}{ FIELDS } & \multirow{2}{*}{ EINGESTATES } & \multicolumn{4}{|c|}{ MASSES SQUARED } \\
\cline { 3 - 6 } & & SYMBOL & $K=K_{1}$ & $K=K_{2}$ & $K=K_{i+2}$ \\
\hline 2 real scalars & $\widehat{\theta}$ & $\widehat{m}_{\theta}^{2}$ & $4 \widehat{H}_{\mathrm{CI}}^{2}$ & \multicolumn{2}{|c|}{$6 \widehat{H}_{\mathrm{CI}}^{2}$} \\
\cline { 3 - 6 } 1 complex scalar & $\widehat{s}, \widehat{\bar{s}}$ & $\widehat{m}_{s}^{2}$ & $6\left(2 k_{S} f_{R}-1 / 3\right) \widehat{H}_{\mathrm{CI}}^{2}$ & $12 k_{S} \widehat{H}_{\mathrm{CI}}^{2}$ \\
\hline 4 Weyl spinors & $\widehat{\psi}_{ \pm}$ & $\widehat{m}_{\psi \pm}^{2}$ & \multicolumn{3}{|c|}{$3 n^{2} \widehat{H}_{\mathrm{CI}}^{2} / 2 c_{\mathrm{K}} \phi^{2} f_{R}^{1+m}$} \\
\hline
\end{tabular}

Table 2: Mass-squared spectrum for $K=K_{i}$ and $K=K_{i+2}(i=1,2)$ along the path in Eq. (2.2).

Note that for $m=0$ [ $m=1], F_{R}$ and $F_{\mathrm{K}}$ in $K_{1}$ and $K_{3}\left[K_{2}\right.$ and $\left.K_{4}\right]$ are totally decoupled, i.e. no higher order term is needed. Also we use only integer prefactors for the logarithms avoiding thereby any relevant tuning - cf. Ref. [10]. Our models, for $c_{\mathrm{K}} \gg c_{R}$, are completely natural in the 't Hooft sense because, in the limits $c_{R} \rightarrow 0$ and $\lambda \rightarrow 0$, the theory enjoys the enhanced symmetries

$$
\Phi \rightarrow \Phi^{*}, \Phi \rightarrow \Phi+c \text { and } S \rightarrow e^{i \alpha} S,
$$

where $c$ is a real number. It is evident that our proposal is realized more attractively within SUGRA than within the non-SUSY set-up, since both $f_{\mathrm{K}}$ and $f_{R}$ originate from the same function $K$.

To verify the appropriateness of $K$ 's in Eqs. (L.7ad) - (L.7d), we can first remark that, along the trough in Eq. (․2), these are diagonal with non-vanishing elements $K_{S S^{*}}$ and $K_{\Phi \Phi^{*}}=J^{2}$, where $J$

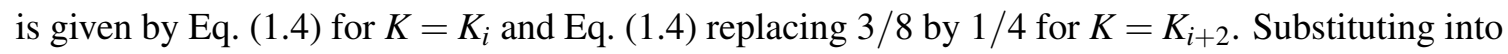
Eq. (2.5) $K^{S S^{*}}=1 / K_{S S^{*}}$ and $\exp K=1 / f_{R}^{N}$, where

$$
K_{S S^{*}}=\left\{\begin{array}{l}
1 / f_{R} \\
1
\end{array} \text { and } N=\left\{\begin{array}{l}
3 \\
2
\end{array} \text { for } K=\left\{\begin{array}{l}
K_{i} \\
K_{i+2}
\end{array} \text { with } i=1,2,\right.\right.\right.
$$

we easily deduce that $\widehat{V}_{\mathrm{CI}}$ in Eq. (L.4) is recovered. If we perform the inverse of the conformal transformation described in Eqs. (ㅍ.3al) and (ㄷ.2) with frame function $\Omega / N=-e^{-K / N}$ we can easily show that $f_{R}=-\Omega / N$ along the path in Eq. (L2). Note, finally, that the conventional Einstein gravity is recovered at the SUSY vacuum, $\langle S\rangle=\langle\Phi\rangle=0$, since $\left\langle f_{R}\right\rangle \simeq 1$.

Defining the canonically normalized fields via the relations $d \widehat{\phi} / d \phi=\sqrt{K_{\Phi \Phi^{*}}}=J, \widehat{\theta}=J \theta \phi$ and $(\widehat{s}, \widehat{\bar{s}})=\sqrt{K_{S S^{*}}}(s, \bar{s})$ we can verify that the configuration in Eq. (․2.) is stable w.r.t the excitations of the non-inflaton fields. Taking the limit $c_{\mathrm{K}} \gg c_{R}$ we find the expressions of the masses squared $\widehat{m}_{\chi^{\alpha}}^{2}$ (with $\chi^{\alpha}=\theta$ and $s$ ) arranged in Table $\square$, which approach rather well the quite lengthy, exact formulas. From these expressions we appreciate the role of $k_{S}>0$ in retaining positive $\widehat{m}_{s}^{2}$. Also we confirm that $\widehat{m}_{\chi^{\alpha}}^{2} \gg \widehat{H}_{\mathrm{CI}}^{2}=\widehat{V}_{\mathrm{CI} 0} / 3$ for $\phi_{\mathrm{f}} \leq \phi \leq \phi_{\star}$. In Table \ we display the masses $\widehat{m}_{\psi^{ \pm}}^{2}$ of the corresponding fermions too with eignestates $\widehat{\psi}_{ \pm}=\left(\widehat{\psi}_{\Phi} \pm \widehat{\psi}_{S}\right) / \sqrt{2}$, defined in terms of $\widehat{\psi}_{S}=$ $\sqrt{K_{S S^{*}}} \psi_{S}$ and $\widehat{\psi}_{\Phi}=\sqrt{K_{\Phi \Phi^{*}}} \psi_{\Phi}$, where $\psi_{\Phi}$ and $\psi_{S}$ are the Weyl spinors associated with $S$ and $\Phi$ respectively. Note, finally, that $\widehat{m}_{\chi^{\alpha}} \ll m_{\mathrm{P}}$, for any $\chi^{\alpha}$, contrary to similar cases [11] where the inflaton belongs to gauge non-singlet superfields.

Inserting the derived mass spectrum in the well-known Coleman-Weinberg formula, we can find the one-loop radiative corrections, $\Delta \widehat{V}_{\mathrm{CI}}$ to $\widehat{V}_{\mathrm{CI}}$. It can be verified that our results are immune from $\Delta \widehat{V}_{\mathrm{CI}}$, provided that the renormalization group mass scale $\Lambda$, is determined conveniently and $k_{S \Phi}$ and $k_{S}$ are confined to values of order unity. 


\section{Results}

The present inflationary scenario depends on the parameters: $n, m, r_{R \mathrm{~K}}, \lambda / c_{\mathrm{K}}^{n / 4}$. Note that the two last combinations of parameters above replace $c_{\mathrm{K}}, c_{R}$ and $\lambda$. This is because, if we perform a rescaling $\phi=\tilde{\phi} / \sqrt{c_{\mathrm{K}}}$, Eq. ([.2) preserves its form replacing $\phi$ with $\tilde{\phi}$ and $f_{\mathrm{K}}$ with $f_{R}^{m}$ where $f_{R}$ and $V_{\mathrm{CI}}$ take, respectively, the forms

$$
f_{R}=1+r_{R \mathrm{~K}} \tilde{\phi}^{n / 2} \text { and } V_{\mathrm{CI}}=\lambda^{2} \tilde{\phi}^{n} / 2^{n / 2} c_{\mathrm{K}}^{n / 2},
$$

which, indeed, depend only on $r_{R K}$ and $\lambda^{2} / c_{\mathrm{K}}^{n / 2}$. Imposing the restrictions of Sec. ए.2 we can delineate the allowed region of these parameters. Below we first extract some analytic expressions - see Sec. B.]- which assist us to interpret the exact numerical results presented in Sec. [.2.2.

\subsection{Analytic Results}

Assuming $c_{\mathrm{K}} \gg c_{R}$, Eq. ([ए.36) yields $J \simeq \sqrt{c_{\mathrm{K}}} / f_{R}^{(1-m) / 2}$. Inserting the last one and $\widehat{V}_{\mathrm{CI}}$ from Eq. (메) in Eq. (ㅁ. $)$ we extract the slow-roll parameters for this model as follows - cf. Eq. (매):

$$
\widehat{\varepsilon}=n^{2} / 2 \phi^{2} c_{\mathrm{K}} f_{R}^{1+m} \text { and } \widehat{\eta}=2(1-1 / n) \widehat{\varepsilon}-(4+n(1+m)) c_{R} \phi^{n / 2} \widehat{\varepsilon} / 2 n .
$$

Given that $\phi \ll 1$ and so $f_{R} \simeq 1$, nMI terminates for $\phi=\phi_{\mathrm{f}}$ found by the condition

$$
\phi_{\mathrm{f}} \simeq \max \left\{n / \sqrt{2 c_{\mathrm{K}}}, \sqrt{(n-1) n / c_{\mathrm{K}}}\right\},
$$

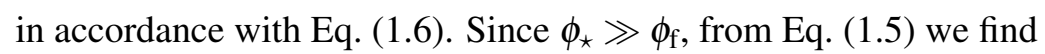

$$
\widehat{N}_{\star}=\frac{c_{\mathrm{K}} \phi_{\star}^{2}}{2 n}{ }_{2} F_{1}\left(-m, 4 / n ; 1+4 / n ;-c_{R} \phi_{\star}^{n / 2}\right)= \begin{cases}c_{\mathrm{K}} \phi_{\star}^{2} / 2 n & \text { for } m=0, \\ \left(f_{R}^{1+m}-1\right) / 8(1+m) r_{R \mathrm{~K}} & \text { for } n=4,\end{cases}
$$

where ${ }_{2} F_{1}$ is the Gauss hypergeometric function. Concentrating on the cases with $m=0$ or $n=4$, we solve Eq. (B.4) w.r.t $\phi_{\star}$ with results

$$
\phi_{\star} \simeq \begin{cases}\sqrt{2 n \widehat{N}_{\star} / c_{\mathrm{K}}} & \text { for } m=0, \\ \sqrt{f_{m \star}-1 / \sqrt{r_{R K} c_{\mathrm{K}}}} & \text { for } n=4,\end{cases}
$$

where $f_{m \star}^{1+m}=1+8(m+1) r_{R K} \widehat{N}_{\star}$. In both cases there is a lower bound on $c_{\mathrm{K}}$, above which $\phi_{\star}<1$ and so, our proposal can be stabilized against corrections from higher order terms - e.g., for $n=$ $4, m=1$ and $r_{R \mathrm{~K}}=0.03$ we obtain $140 \lesssim c_{\mathrm{K}} \lesssim 1.4 \cdot 10^{6}$ for $3.3 \cdot 10^{-4} \lesssim \lambda \lesssim 3.5$. The correlation

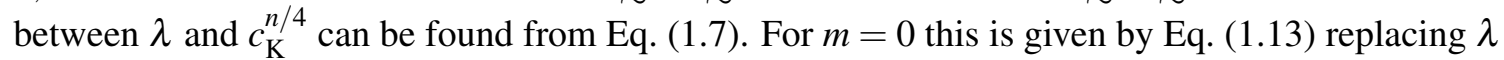
with $\lambda / c_{\mathrm{K}}^{n / 4}$ and $c_{R}$ with $r_{R \mathrm{~K}}$ in the definition of $f_{n \star}$. For $n=4$ we obtain

$$
\lambda=16 \sqrt{3 A_{\mathrm{s}}} \pi c_{\mathrm{K}} r_{R \mathrm{~K}}^{3 / 2} /\left(f_{m \star}-1\right)^{3 / 2} f_{m \star}^{(1+m) / 2} .
$$

As regards the inflationary observables, these are obviously given by Eqs. (‥14al) and ([..46) for the trivial case with $m=0$. For $m \neq 0$, however, these are heavily altered. In particular, for $n=4$ we obtain

$$
\begin{aligned}
n_{\mathrm{S}} & =1-8 r_{R K} \frac{m-1-(m+2) f_{m \star}}{\left(f_{m \star}-1\right) f_{m \star}^{1+m}}, r=\frac{128 r_{R \mathrm{~K}}}{\left(f_{m \star}-1\right) f_{m \star}^{1+m}}, \\
a_{\mathrm{S}} & =\frac{64 r_{R \mathrm{~K}}^{2}(1+m)(m+2)}{\left(f_{m \star}-1\right)^{2} f_{m \star}^{4(1+m)}} f_{m \star}^{2}\left(f_{m \star}^{2 m}\left(\frac{1-m}{m+2}+\frac{2 m-1}{m+1} f_{m \star}\right)-f_{m \star}^{2(1+m)}\right) .
\end{aligned}
$$

The formulae above is valid only for $r_{R K}>0$ - see Eq. (B.5) - and is simplified [1] for low $m$ 's. 

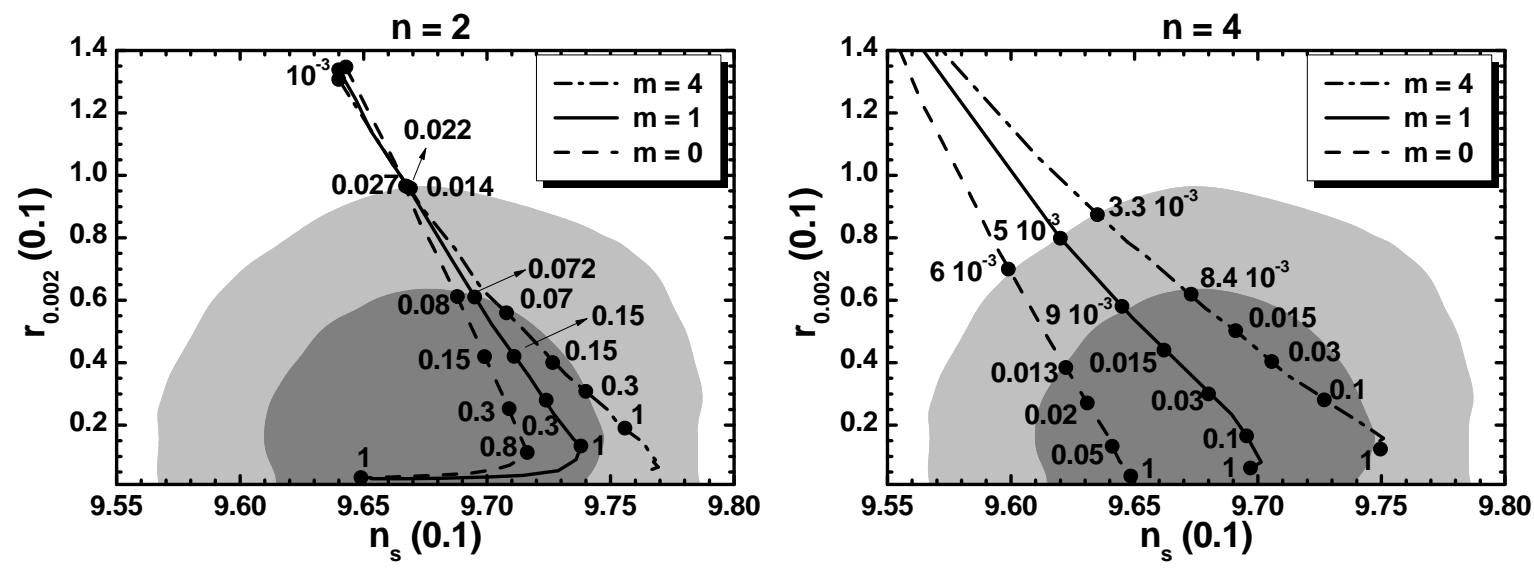

Figure 1: Allowed curves in the $n_{\mathrm{s}}-r_{0.002}$ plane for $n=2$ and $4, m=0$ (dashed lines), $m=1$ (solid lines), $m=4$ (dot-dashed lines), and various $r_{R K}$ 's indicated on the curves. The marginalized joint $68 \%$ [95\%] regions from Planck, BICEP2/Keck Array and BAO data are depicted by the dark [light] shaded contours.

\subsection{Numerical Results}

The conclusions obtained in Sec. B.J can be verified and extended to others $n$ 's and $m$ 's nu-

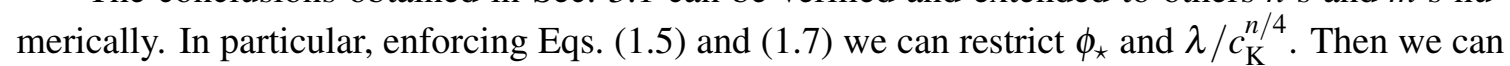
compute the model predictions via Eq. ([L.8), for any selected $m, n$ and $r_{R K}$. The outputs, encoded as lines in the $n_{\mathrm{s}}-r_{0.002}$ plane, are compared against the observational data $[7,8]$ in Fig. $\mathbb{W}$ for $n=2$ (left panel) and 4 (right panel) setting $m=0,1$ and 4 - dashed, solid, and dot-dashed lines respectively. The variation of $r_{R \mathrm{~K}}$ is shown along each line. To obtain an accurate comparison, we compute $r_{0.002}=16 \widehat{\varepsilon}\left(\phi_{0.002}\right)$ where $\phi_{0.002}$ is the value of $\phi$ when the scale $k=0.002 / \mathrm{Mpc}$, which undergoes $\widehat{N}_{0.002}=\left(\widehat{N}_{\star}+3.22\right)$ e-foldings during nMI, crosses the inflationary horizon.

From the plots in Fig. W we observe that, for low enough $r_{R K}$ 's - i.e. $r_{R K}=10^{-4}$ and 0.001 for $n=4$ and $2-$, the various lines converge to the $\left(n_{\mathrm{s}}, r_{0.002}\right)$ 's obtained within the simplest models of chaotic inflation with the same $n$. At the other end, the lines for $n=4$ terminate for $r_{R \mathrm{~K}}=1$, beyond which the theory ceases to be unitarity safe - as anticipated in Sec. ㄸ. 4 - whereas the $n=2$ lines approach an attractor value, comparable with the value in Eq. (미), for any $m$.

For $m=0$ we reveal the results of Sec. ㄴ.3, i.e. the displayed lines are almost parallel for $r_{0.002} \geq 0.02$ and converge at the values in Eq. (‥19) - for $n=4$ this is reached even for $r_{R K}=1$.

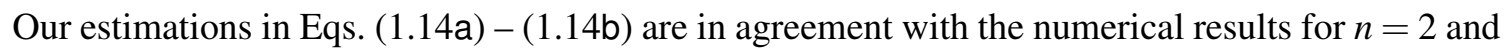
$r_{R \mathrm{~K}} \lesssim 1$ or $n=4$ and $r_{R \mathrm{~K}} \lesssim 0.05$. We observe that the $n=2$ line is closer to the central values in Eq. (ㅁ.9) whereas the $n=4$ one deviates from those.

For $m>0$ the curves change slopes w.r.t to those with $m=0$ and move to the right. As a consequence, for $n=4$ they span densely the 1- $\sigma$ ranges in Eq. (ㅍ. ) for quite natural $r_{R K}$ 's - e.g. $0.005 \lesssim r_{R \mathrm{~K}} \lesssim 0.1$ for $m=1$. It is worth mentioning that the requirement $r_{R \mathrm{~K}} \leq 1$ (for $n=4$ ) provides a lower bound on $r_{0.002}$, which ranges from 0.004 for $m=0$ to 0.015 (for $m=$ 4). Therefore, our results are testable in the forthcoming experiments [12] hunting for primordial gravitational waves. Note, finally, that our findings in Eqs. (3.7a) - (B.7b) approximate fairly the numerical outputs for $0.003 \lesssim r_{R K} \leq 1$. 


\section{Conclusions}

We reviewed the implementation of kinetically modified $\mathrm{nMI}$ in both a non-SUSY and a SUSY framework. The models are tied to the potential $V_{\mathrm{CI}}$ and the coupling function of the inflaton to gravity given in Eq. (메) and the non-canonical kinetic mixing in Eq. (ㄴ.24). This setting can be elegantly implemented in SUGRA too, employing the super-and Kähler potentials given

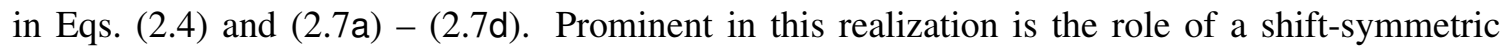
quadratic function $F_{\mathrm{K}}$ in Eq. (12.6) which remains invisible in the SUGRA scalar potential while dominates the canonical normalization of the inflaton. Using $m \geq 0$ and confining $r_{R \mathrm{~K}}$ to the range $\left(3.3 \cdot 10^{-3}-1\right)$, where the upper bound does not apply to the $n=2$ case, we achieved observational predictions which may be tested in the near future and converge towards the "sweet" spot of the present data - especially for $n=4$. These solutions can be attained even with subplanckian values of the inflaton requiring large $c_{\mathrm{K}}$ 's and without causing any problem with the perturbative unitarity. It is gratifying, finally, that the most promising case of our proposal with $n=4$ can be studied analytically and rather accurately.

\section{Acknowledgments}

This research was supported from the MEC and FEDER (EC) grants FPA2011-23596 and the Generalitat Valenciana under grant PROMETEOII/2013/017.

\section{References}

[1] C. Pallis, Phys. Rev. D 91, 123508 (2015) [arXiv: 1503.05887 ].

[2] D. S. Salopek, J. R. Bond and J.M. Bardeen, Phys. Rev. D 40, 1753 (1989); F.L. Bezrukov and M. Shaposhnikov, Phys. Lett. B 659, 703 (2008) [arXiv: 0710.3755 ].

[3] C. Pallis, Phys. Lett. B 692, 287 (2010) [arXiv: 1002 . 4765];

C. Pallis and Q. Shafi, Phys. Rev. D 86, 023523 (2012) [arXiv: 1204 . 0252].

[4] R. Kallosh, A. Linde, and D. Roest, Phys. Rev. Lett. 112, 011303 (2014) [arXiv: 1310 . 3950].

[5] J.L.F. Barbon and J.R. Espinosa, Phys. Rev. D 79, 081302 (2009) [arXiv: 0903.0355 ]; C.P. Burgess, H.M. Lee, and M. Trott, JHEP 07, 007 (2010) [arXiv:1002 .2730].

[6] A. Kehagias, A.M. Dizgah, and A. Riotto, Phys. Rev. D 89, 043527 (2014) [arXiv:1312 .1155].

[7] Planck Collaboration, arXiv:1502.02114.

[8] P.A.R. Ade et al., Phys. Rev. Lett. 114, 101301 (2015) [arXiv:1502.00612].

[9] M.B. Einhorn and D.R.T. Jones, JHEP 03, 026 (2010) [arXiv: 0912 .2718];

H.M. Lee, JCAP 08, 003 (2010) [arXiv: 1005 .2735];

S. Ferrara et al., Phys. Rev. D 83, 025008 (2011) [arXiv: 1008.2942];

C. Pallis and N. Toumbas, JCAP 02, 019 (2011) [arXiv:1101.0325].

[10] C. Pallis, JCAP 10, 058 (2014) [arXiv:1407.8522];

C. Pallis and Q. Shafi, JCAP 03, 023 (2015) [arXiv:1412.3757].

[11] G. Lazarides and C. Pallis, arXiv:1508.06682;

C. Pallis, to appear.

[12] P. Creminelli et al., arXiv:1502.01983. 